\title{
Nachruf auf Fritz H. Kemper
}

26 Jahre lang war Professor Dr. med. Dr. h.c. mult. Fritz H. Kemper Herausgeber der Zeitschrift für Phytotherapie. Eine bemerkenswerte Konstante. Gemeinsam mit Professor Franz-C. Czygan hat er im Jahr 1985 die ZPT von ihrem Gründer und Autor des Standardwerkes „Lehrbuch der Phytotherapie“, Rudolf F. Weiß, übernommen. Dieser - damals ebenfalls 90 Jahre alt - hatte ihnen seine Bitte zu „Mut und Beharrlichkeit gegenüber den zunehmend dirigistischen Tendenzen im Gesundheitswesen“ mit auf den Weg gegeben.

Lange haben die beiden nicht hinsichtlich der Zusage überlegen müssen. Der besondere Stellenwert von Arzneipflanzen war Kern ihrer jeweiligen Profession - und die Möglichkeit, ein wissenschaftliches Periodikum zu gestalten, schien ihnen nicht nur notwendig, sondern reizvoll. Was zunächst auf Wunsch von Kemper und Czygan auf drei Jahre begrenzt sein sollte, hat ihnen offensichtlich so viel Freude bereitet, dass sie Heft um Heft konzipierten und zahlreiche Autoren zur Mitarbeit gewinnen konnten. Ein großes Anliegen war ihnen die Publikation evidenzbasierter Phytotherapie bereits zu einer Zeit, als es diesen Begriff noch gar nicht gab. Dass dies über einen so langen Zeitraum gelingen konnte, zeigt, wieviel Potenzial beide in dem Thema gesehen haben.

Stets im Blick von Professor Kemper stand die Etablierung der wissenschaftlichen Phytotherapie als selbstverständlichem Teil der Schulmedizin. Das findet sich auch in seinen - seltenen - Editorials, die schon aufgrund der sprachlichen Prägnanz lesenswert waren. Sie beschäftigten sich meist mit der deutschen und europäischen Gesundheitspolitik. An seinem Engagement in einer Vielzahl von Gremien hat er die Leser teilhaben lassen. Wir konnten am Beispiel der Arzneipflanzen viel über die Komplexität der europäischen Harmonisierung erfahren. Ein langer Weg, der aus deutscher Sicht nicht nur Befürworter hatte. Professor Kemper sah darin neben der Notwendigkeit auch die Chance. Nicht zuletzt deshalb war ihm die Arbeit der ESCOP so wichtig. Den Georg Thieme Verlag konnte er von der internationalen Publikation der ESCOP-Monografien 2003 und 2009 überzeugen.

Nicht verhindern konnte er - ausgerechnet zum 25-jährigen Jubiläum der ZPT - das GKV-Modernisierungsgesetz im Jahr 2004 und damit den Ausschluss der meisten pflanzlichen Arzneimittel aus der Erstattungsfähigkeit. Die gemeinsamen Anstrengungen mit Professor Rudolf Bauer von der Gesellschaft für Arzneipflanzenforschung und Professor Ivar Roots von der Deutschen Gesellschaft für Klinische Pharmakologie und Therapie blieben ohne Wirkung. Umso mehr konnte er sich 2013 über die Ernte der langjährigen gemeinsamen Arbeit mit Frau Professor Kraft freuen, nämlich die Aufnahme der Gesellschaft für Phytotherapie in die AWMF.

Unvergessen sind die Telefonate mit ihm. Wenn man ihn, den Vielgereisten, dann tatsächlich mal im Münsteraner Institut in der Domagkstraße erreichte, gelang dies vor allem dank seiner Sekretärin, Frau Müller. Fast immer gab es dabei eine praktikable Lösung für die Nöte des ZPT-Redakteurs und darüber hinaus die neuesten Informationen zu Phytoforschung und Phytopolitik. Tempi passati.

\section{Hanno Kretschmer \\ Zeitschrift für Phytotherapie \\ Georg Thieme Verlag}

